\title{
CATASTROFISMO VERSUS ACTUALISMO. IMPLICACIONES DIDÁCTICAS
}

\author{
PEDRINACI, E. \\ Instituto Andaluz de Formación del Profesorado. Sevilla.
}

\section{SUMMARY}

This paper analises the historic evolution of the «actualist» and «catastrophist» geologic interpretations, describes possible epistemologic obstacles and considers their treatment in the Secondary School.

«...pero, reconocer la mutabilidad de la Tierra, de Ios seres vivientes que han habitado en ella y hasta de Ios Cielos mismos, es algo que los hombres sólo hacen bajo la presión de argumentos abrumadores».

Toulmin 1968 «The Discovery of Time»

\section{INTRODUCCIÓN}

Pocos pasajes de Ia historia de la Geología son tan apasionantes como el de la controversia que tuvo lugar a finales deI siglo XVIII y, muy especialmente, en el primer tercio del XIX, entre Ios partidarios del Uniformitarismo y los del Catastrofismo. Pero, como se pretende mostrar en este trabajo, el debate no se limita a esa época (simplificación, por otra parte, muy frecuente), sino que se remonta a la antiguiedad clásica y ha resurgido en nuestros días.

La historia de la ciencia puede aportar informaciones relevantes acerca del problema de la construcción del conocimiento (Saltiel y Viennot 1985, Gagliardi y Giordan 1986). Es una herramienta útil para definir los contenidos fundamentales de la enseñanza, facilita la detección de obstáculos epistemológicos y puede orientar sobre posibles modos de tratamiento (Sequeiros 1981, Pedrinaci 1991).

Si analizamos los conceptos implicados en el debate Actualismo/Catastrofismo, Ios obstáculos que en la construcción de dichos conceptos parecen haber existido y los métodos de acercamiento e interpretación de la realidad que utilizaron, se comprenderán muchas de las dificultades que presentan para nuestros alumnos y la importancia que tienen en el aprendizaje de la Geología.

\section{CLARIFICACIÓN TERMINOLÓGICA}

Los términos Catastrofismo, Uniformitarismo y Actualismo no siempre han sido utilizados con un mismo significado en la literatura geológica. El confusionismo se debe, de una parte, a que, entre las perspectivas catastrofistas y uniformitaristas más extremas, ha habido numerosas posiciones intermedias que recogían elementos de ambas; $y$, de otra, a que en el continente europeo se denominó Actualismo a lo que los geólogos de habla inglesa llamaban «uniformitarianism». Como consecuencia, ha ocurrido to que Anguita (1988) califica de «interminable guerra de palabras».

Para una correcta comprensión de lo que aquí se dice puede ser, en consecuencia, conveniente señalar a qué conceptos nos estamos refiriendo. El Catastrofismo, en su significado clásico, es aquella teoría que pretende explicar los fenómenos geológicos como consecuencia de grandes catástrofes ocurridas en el pasado de la Tierra (el diluvio sería un claro ejemplo); considera que los procesos que ocurren en la actualidad son manifiestamente insuficientes para explicar las grandes estructuras geo- 
lógicas. El Uniformitarismo defiende que los procesos geológicos ocurridos en el pasado son los mismos que operan en la actualidad y han actuado con el mismo grado y energía que lo hacen hoy.

Desde esta definición, un tanto arquetípica y sin duda simplificadora, se trataría de dos teorías en las que subyacen concepciones diferentes del mundo, e incluso de la vida y del hombre. Una interpreta los cambios como grandes revoluciones en el globo; la otra, como resultado de pequeñas modificaciones acumuladas a lo largo del tiempo.

EI Actualismo, como se ha reseñado, ha sido utilizado como sinónimo de Uniformitarismo. Sin embargo, en to que a nosotros respecta y de acuerdo con Hooykaas (1970), consideramos que el Actualismo no es necesariamente gradualista; es decir, no exige un ritmo idéntico de los procesos naturales para todo el pasado geológico. Sólo defiende que la forma en que podemos reconstruir el pasado es analizando las causas que intervienen en la actualidad. Se puede decir, en consecuencia, que todo unfformitarista es actualista, pero no a la inversa.

En síntesis, el Catastrofismo y el Uniformitarismo serían teorías de la Tierra, en tanto que el Actualismo sería un método de análisis.

\section{UNA HISTORIA CONTROVERTIDA Y RE- CURRENTE}

La Geología como ciencia ha sido de muy tardía aparición. Sin embargo, la observación y el tratamiento de problemas que hoy no dudaríamos en calificar de geológicos es tan antigua como la historia del hombre.

En efecto, en el antiguo Egipto se creía (Cailieux 1961) en un pasado dividido en ciclos, cuya duración era cifrada por algunos en 120.000 años, en tanto que otros los ampliaban hasta 360.000 años. La separación entre uno y otro ciclo estaba reguiada por los astros y era determinada por catástrofes, como grandes diluvios o conflagraciones.

Probablemente sea Heródoto (siglo $\mathrm{V} \mathrm{aC}$ ) quien hace la primera descripción de un proceso geológico. Estudiando el valle del Nilo concluye que ha debido ser en otra época un golfo que, gracias a las aportaciones del río, se ha ido colmatando hasta transformarse en tierra firme. En sus observaciones sigue procedimientos que no sería exagerado calificar de actualistas. Incluso sugiere (Historias, II, 10 ss) la posibilidad de que en unos veinte mil años ocurra un proceso de colmatación similar en el mar Rojo (en el supuesto de que el Nilo modificase su curso y desembocase en este mar). Al margen de lo desacertada que fuese su predicción (especialmente si tenemos en cuenta, a la luz de la tectónica de placas, la génesis y evolución del mar Rojo), tiene el enorme interés de que, desde la observación de lo que ocurre en el presente, hace una doble extrapolación de una parte hacia el pasado, señalando cómo habría sido el actual valle del
Nilo, y, de otra, hacia el futuro (siempre muy arriesgado en toda ciencia histórica), prediciendo to que puede ocurrir en otra zona a la que considera similar.

Aristóteles (384-322 aC) hace en Los Meteoros algunas reflexiones geológicas importantes que tendrán, como casi todo su saber, gran influencia posterior:

«Los mismos lugares de la tierra no siempre son húmedos o secos sino que cambian según la aparición y la desaparición de los ríos; por eso también se producen cambios en la disposición de la tierra firme y del mar, y tanto la tierra como el mar no permanecen siempre iguales, sino que se encuentra el mar allí donde estaba la tierra firme y allí donde ahora se encuentra el mar habrá de nuevo una tierra. Y debemos pensar que estos hechos se producen según un cierto orden y una cierta periodicidad [...] Debido a que toda la evolución de la tierra se produce gradualmente y en tiempos muy largos comparándolos con nuestra vida, estos cambios escapan a la observación..." (Meteoros, I, 14, 351. El subrayado es nuestro).

Aristóteles defiende que los procesos ordinarios producen importantes efectos como consecuencia de su acción continuada en largos períodos de tiempo. Su visión del universo como algo eterno le elimina uno de los obstáculos con los que, como se verá, han tropezado otras interpretaciones, al tener que explicar grandes cambios en cortos espacios de tiempo. Pero, simultáneamente, le genera otro derivado de la concepción de la eternidad del mundo que Teofrasto tratará de evidenciar utilizando argumentos geodinámicos y que Aristóteles resuelve con su visión cíclica de la Tíerra. Una buena muestra de ello es el siguiente texto (Duhem 1959) en el que Filón de Alejandría parece recoger las ideas de Teofrasto:

«Si la Tierra no hubiera tenido un principio, ninguna de sus partes aparecería hoy más alta que las demás; ya todos los montes habrían sido allanados, todas las colinas reducidas al mismo nivel que las Ilanuras. Pensemos, realmente, en las innumerables Iluvias anuales que habrían caído desde toda la eternidad; se comprenderá que entre los lugares que se elevan, unos habrían sido, sin duda alguna, ró́dos y arrastrados por los torrentes; otros se habrían derrumbado por su propio peso, de tal manera que la tierra que los formaba se hallaría, ahora, repartida uniformemente por todas partes y perfectamente allanada.»

Como vemos, se trata de un texto claramente actualista, que participa de una visión unidireccional de los procesos geológicos, en el que siguiendo una causalidad simple, ante la ausencia de mecanismos creadores de relieve, los procesos de erosión y sedimentación deberían imponer sư lógica niveladora.

¿Quiere todo ello decir que en la Grecia clásica las tesis dominantes cran actualistas? La respuesta no puede ser categórica ya que, junto a textos como los reseñados existen otros que recurren a interpretaciones catastrofistas con frecuencia ligadas a seísmos y que provocan hundimientos de montañas e incluso de regiones enteras. Así, por ejemplo, Platón (427-348 aC), en algunos de sus 
últimos diúlogos, como Timeo o Kritias, hace referencia a estas cuestiones y atribuye a un violento seísmo el hundimiento de la Atlántida.

En la mitología griega (como en la hebrea, la egipcia, la hindú o la china) existe la tradición de un gran diluvio (Táton 1964), pero en contra de lo que ocurrirá con la religión cristiana (especialmente con la protestante) su incidencia en las interpretaciones de la historia de la Tierra va a ser muy escasa.

Un buen ejemplo de lo que se acaba de indicar se puede encontrar en Ovidio (43-17 aC). Así, en algunos de los poemas de Las Metamorfosis (266 y 267) hace una excelente descripción actualista de procesos erosivos de montañas, formación de valles o cambios en la distribución de tierras y mares. Mientras que en otros poemas (296306) recurre a explicaciones míticas de «la fuerza terrible de los vientos encerrados en ciegas cavernas" para justificar el origen de las montañas (idea, por otra parte, muy frecuente en la antiguiedad y que encontraremos aún en Kircher en el siglo XVII).

Un caso, en cierto sentido, análogo es el de Séneca (4-65 $\mathrm{aC})$, quien en sus Cuestiones Naturales hace reflexiones tan actualistas como «buscamos la causa natural que actúa constantemente y no por excepción y fortuitamente» (II, LV, 3), pero también otras más próximas a posiciones catastrofistas: «La naturaleza... para dar nacimiento a las cosas economiza sus fuerzas, se regula ella misma y procede por crecimientos insensibles. Para destruirlas, actúa bruscamente y con toda su violencia».

$\Lambda$ partir del siglo IV de nuestra era, y durante un largo período van a ser dominantes las interpretaciones catastrofistas. Por ejemplo Lactancio (siglo IV dC) en Divinas Instituciones:

«Pođría enumerar cuántas veces, sacudidas por movimientos súbitos, se abrieron las tierras, o descendieron a los abismos; cuántas veces, hundiđas en las aguas, ciưades e islas desaparecieron en las profundidades; los pantanos sumergieron los campos fértiles, los ríos y las aguas estancadas se desecaron, los montes cayeron a pedazos o se nivelaron con las llanuras; y además, el fuego innato, escondido, consumió muchas regiones y muchos cimientos de montañas" (citado por Ellenberger 1988).

Igual ocurre con San Agustín (354-430), quien describe una amplia gama de cambios catastróficos (tomados de Apuleyo) que sufren las tierras: diluvios, hundimientos, conftagraciones... O con Arnobio (siglo V), o san Isidoro de Sevilla (560-636) (Crombie 1959).

Entramos ya en una época en la que se va a dejar notar la influencia de las interpretaciones que de la Biblia se hacen; especialmente en dos aspectos: de una parte, el diluvio, que se utilizará para explicar los más variados procesos (por ejemplo, la existencia de fósiles marinos en zonas alejadas del mar); y, de otra, la «cronología corta» o cronología bública que, al asignarle a ta Tierra una existencia anterior muy breve, obliga a buscar mecanismos que puedan provocar importantes efectos en un tiempo muy corto (catástrofes).
Los árabes recogen la trađición griega y hacen algunas importantes aportaciones. Así, los Hermanos de la $\mathrm{Pu}+$ reza y de la Sinceridad (lkhwân al-Safâ, siglo X) describen algo que nos recuerda el ciclo huttoniano y los principios de superposición de Steno (Duhem 1959). También Avicena (990-1037) y Averroes (1126-1198) hacen interesantes aportaciones en la misma línea del pensamiento aristotélico.

Planteamientos similares a éstos se encuentran posteriormente en el occidente cristiano. Es el caso de Alberto Magno (1206-1280), de Jean Buridan (1300-1358) o de Alberto de Sajonia, entre otros. Para ellos el tiempo es ilimitado y el universo estable. En la Tierra se producen cambios lentos y graduales.

A diferencia de Io que ocurre en otras ciencias y, sobre todo, en las artes, el Renacimiento realiza una escasa aportación a la Geología, incluso sufre un retroceso (si se compara con los Hermanos de la Pureza o con Jean Buridan), en buena parte condicionado porque se alcanza el apogeo de la "cronología corta» y se desprecian las aportaciones de los últimos neoaristotelistas medievales.

Una excepción a esa situación es Leonardo da Vinci (1452-1519), quien dejó una importante cantidad de observaciones geológicas, aunque repartidas de una manera bastante desordenada por sus manuscritos; 10 que, unido a que no se editaran, hizo que su influencia fuese escasa y tardía. Se suelen referir sus estudios a la naturaleza y el origen de los fósiles (cuestion ésta que será el problema geológico central durante los siglos XVI y XVII), pero, probablemente, no sea lo más interesante e innovador de sus trabajos. En ellos, sin llegar a construir una «teoría de la Tierra», se ofrece una imagen de conjunto de los fenómenos terrestres. Hace observaciones sobre los procesos de erosión y sedimentación, sobre el origen de los valles y de las montañas, y asume, de manera implícita, una larga duración de los tiempos.

Es necesario mencionar un diluvialista radical que, probablemente, tuvo gran influencia posterior: Martin Lutero quien en su obra In primum librum Mose enarrationes (1544) comenta que «después deI Diluvio se encuentran montañas allí donde antes había campos en una agradable llanura; igualmente no hay duda de que hay ahora fuentes donde antes no había ninguna y viceversa. En efecto, cambió toda la faz de la tierra». Para Ellenberger (1988), en los textos de Lutero se encuentra una de las fuentes teóricas que utilizarán Ios diluvialistas protestantes, especialmente los ingleses y suizos, siglo y medio más tarde, como base de sus teorías de la Tierra.

\section{UN CAMBIO ME'TODOLÓGICO Y CONCEP. TUAL}

En el siglo XVII se producen dos cambios importantes. El primero sería el intento de construir modelos teóricos que pretenden explicar la formación y la estructura de la Tierra. Hasta este momento Ias aportaciones que se han hecho son generalmente dispersas, algunas de ellas nos parecen hoy sorprendentemente certeras, pero, en cual- 
quier caso, no se preocupan de ofrecer un entramado de conceptos y procesos geológicos interconectados. Descartes (1596-1650) es, probablemente, el primero que clabora una verdadera teoría de Ia Tierra (Principia Philosophiae), con aportaciones muy sugerentes sobre cl origen de las montañas (Gohau 1983). Sin embargo sus hipótesis no vienen avaladas por observaciones directas del terreno; metodológicamente son muy poco rigurosas.

El segundo cambio importante se puede calificar, en cierta medida, de metodológico y conceptual (Gil y Carrascosa 1985) y tiene en Steno (Nicis Stensen 1638. 1686) su primer actor. Su contribución le ha hecho merecedor de ser considerado uno de los epadres funđadores» de la Geología. A él debemos la introducción del concepto de estrato y de los principios de continuidad, de horizontalidad, y de superposición de los estratos, que constituyen las bases de la moderna estratigrafía. Tanto en su principal obra, Prodromus, comoen Canis Carchariae, rompe con una constante que presentaban los trabajos geológicos desde la antigüedad clásica y que, de acuerdo con la terminología de Carrascosa y Gil (1985), podríamos denominarla emetodología de la su* perficialidad». Recoge observaciones de la naturaleza y ve en qué medida pueden recibir una explicación saísisfactoria de sus teorías, en qué medida las apoyan o no. Es decir intenta contrastar sus hipótesis utilizando para etlo el gran laboratorio geológico que es la propia naturaleza. Sus interpretaciones son actualistas cuando explica procesos de erosión, de sedimentación, en sus trabajos de crista lografía o, más claramente aún, cuando, intentando deducir «las antiguas caras de la Toscana» (hoy diríamos la historia geológica de la Toscanta), señala con sorprendente lucidez:

«De qué forma las condiciones presentes de una cosa descubren la condición pasada de dicha cosa se pone de manifiesto en Toscana, en la cual las desigualdades de la superficie observadas en su apariencia actual contienen ellas mismas las señales de los diferentes cambios.» (citado por Capel 1987).

Sin embargo recurre a explicaciones que habría que valorar como catastrofistas al tratar problemas tectóni$\cos$.

Pero los trabajos de Steno no forman parte de una comunidad científica que los critique, que los apoye os que profundice en ellos. Se trata de unos trabajos aislados, de tal manera que no es aprovechada su enorme poten. cialidad. Otra de las causas de la escasa influencia de: Steno en sus contemporáneos estaría relacionada, de acuerdo con Ellenberger (1988), con lo enormemente innovadoras, modernas y, consecuentemente, difíciles de asumir que resultaban sus ideas.

Durante el último tercio del siglo XVII y todo el XVIII el diluvio se convierte en el problema geológico central. La frase del Génesis «se rompieron todos los depósitos del gran abismo y se abrieron las cataratas del cielo» (Gen, 7,11, TOB) parecía apoyar tesis catastrofistas. Dichas tesis van a dominar durante todo el siglo XVIII pero se van a ir descargando de su componente mitolo- gico o teológico, de tal manera que, incluso, científicos con visiones experimentalistas recurren a fuerzas enormemente poderosas que existicron en el pasado, pero no en la actualidad, para explicar problemas como el origen de las grandes montañas. Es precisamente esta separación entre los procesos vigentes en el pasado y los actuales lo que caracteriza las posiciones catastrofistas.

Inmediatamente antes de que Yutton (1726-1797) publicara su Theory of the Earth, George H. Toulmin había escrito The Antiquity of the World de enorme similitud. Según McIntyre (1970), es muy probable que Hutton la leyera antes. Toulmin afirma que «la naturaleza siempre es la misma, sus leyes son eternas e inmutables», lo que recuerda que las ideas expuestas en el siguiente pártafo de Hutton (Hallam 1983), en el que se sintetizan los aspectos de su teoría que más interesan para el tema que nos ocupa:

«Si examinamos las cosas actuales, tenemos datos a partir de los cuales podemos razonar sobre lo que fue, $y$, a partir de lo que ya fue, tenemos datos para sacar conclusiones sobre lo que ocurrirá aquí, más adelante. Por consiguiente, partiendo de la suposición de que la labor de la naturaleza es uniforme y constante, encontramos en las apariencias naturales un medio para saber que ha transcurrido una determinada porción de tiempo para producir aquellos acontecimientos de los que hoy vemos los efectos».

Hutton describe también cómo los sedimentos blandos pueden transformarse en rocas, y cómo éstas pueden erosionarse y dar lugar de nuevo a sedimentos. Esa visión cíclica le lleva a pronunciar la famosa frase: «No encontramos huellas de un principio, ni perspectiva de un finals, la cual, por cietto, es muy simitar a otra de Toulmin: «Nunca ha habido un principio, por lo que nunca habrá una conclusión».

J.A. de Luc (1727-1817) polemiza con Hutton y defiende dos eras en la historia de la Tierra; durante la primera se forman los continentes, la segunda comenzaría hace unos cuatro mil años y en clla ya intervienen los mecanismos que conocemos en la actualidad. El límite entre ambas vendría marcado por una gran catástrofe.

Los estudios de paleontología y estratigraffa que realiza Georges Cuvier (1769-1832) le Ilevan a defender en su obra Discours sur les révolutions de la surface du globe que ha habido extinciones masivas de seres vivos, cambios bruscos e importantes del nivel del mar y dislocaciones y vuelcos en los estratos más antiguos que «no dejan lugar a duda de que fueron causas repentinas y violentas las que produjeron las formaciones que observamos hoy». Gracias a los trabajos de Cuvier y de su discípulo L. Elie de Beaumont (1798-1874), entre otros, la teoría catastrofista construye un cuerpo de doctrina que le llevará a dominar el panorama geológico hasta que Charkes Lyell (1797-1875) publica Principles of Geology (1830), obra que adquiere una rápida difusión.

En ella recoge muchas de las ideas que habían sido reiteradamente expuestas, pero las organiza, estructura y fundamenta, presentando el modelo de análisis e inter- 
pretación de los procesos geológicos conocido como Uniformitarismo y caracterizado por: la uniformidad (constancia) en el espacio y el tiempo de las leyes físicas que operan en la naturaleza, la uniformidad de los procesos, y la uniformidad en el ritmo con que han actuado (gradualismo). Esta fase del debate Uniformitarismo/ Catastrofismo es la más conocida y ha sido excelentemente tratada por Hallam (1983), autor al que remitimos.

Ladécadaque sigue a la publicación de Principles of Geology es de una fuerte controversia, sucediéndose argumentos y contraargumentos que acabarán por imponer el sistema uniformitarista.

Como puede verse, la historia la Geología hasta 1830 , con sus dificultades para progresar, sus resistencias, titubeos y contradicciones, responde al comportamiento que Kuhn (1962) describe para el desarrollo de una ciencia hasta que cuenta con su primer paradigma universalmente aceptado.

\section{LNA GEOLOGÍA NEOCATASTROFISTA}

Desputs de Lyell la comunidad científica es uniformitarista. Sin embargo la polémica nunca ha estado completamente cerrada. Durante más de un siglo se ha centrado sobre Ios límites del gradualismo, a veces demasiado estricto, que imponía el Uniformitarismo. En realidad, como señala Kitts (1970), ya el propio Lyell suavizó stu radicalismo inicial y, en una carta a Whewell (1837) escribe :

«Aquella intensidad de la misma o de otras fuerzas terrestres puede ser efectiva; nunca negué su posibilidad, aunque es conjetural. Lamenté que al intentar explicar los fenómenos geológicos, los prejuicios siempre hayan estado del lado malo; siempre ha habido una disposición para razonar a priori sobre la violencia extraordinaria y lo repentino de los cambios, tanto en la corteza terrestre inorgánica comoen los tipos orgánicos, en vez de intentar construir teorías vigorosas de acuerdo con las operaciones ordinarias de la naturalez.a.»

Eil dominio durante más de un siglo de las tesis uniformitaristas hizo, en cierta medida, del Catastrofismo un tabú que ha funcionado como censura (Anguita 1988), o autocensura, de determinadas ideas. En efecto, con frecuencia, las posiciones catastrofistas han sido tildadas de acientíficas; sin embargo, este juicio es, no sólo excesivo, sino probablemente inadecuado, al menos si nos referimos a las posiciones catastrofistas defendidas desde principios del siglo XIX.

En las dos últimas décadas se han acumulado gran número de trabajos que muestran que en el pasado han ocurrido, además de procesos graduales y continuos que pueden ser observados en cualquier momento en la naturaleza, otros esporádicos pero de grandes efectos (cambios climáticos de gran envergadura, cambios en la polaridad del campo magnético, impactos de meteoritos, grandes terremotos...).
Tradicionalmente, las posiciones uniformitaristas han aceptado como causas de los procesos geológicos aquéllas que ocurren en la actualidad, teniendo como unidad de medida del concepto «actualidad» la vida media deI hombre, lo cual, no sólo tiene un dudoso fundamento sino que, además, es manifiestamente insuficiente para unos procesos como los geológicos que, en general, tienen un ritmo y una periodicidad muy superior a esta medida. Por ello hay quienes defienden (Vera 1990) la necesidad de ampliar esta unidad de referencia a todo el Cuaternario.

Para referirse a las catástrofes naturales, como las extinciones en masa (Sequeiros 1990), impactos de grandes meteoritos (Álvarez 1980), importantes cambios eustáticos, 0 , sencillamente grandes inundaciones, se utiliza con frecuencia el término "evento» (Vera 1990), que evita ciertas connotaciones que el catastrofismo ha podido tener y que además presenta una gran similitud ortográfica en diferentes idiomas.

Esta nueva concepción que integra como normales las catástrofes ha sido denominada por Hsu (1983) «Catastrofismo actualista». Otros, como Berggren (1984), prefieren el término «Neouniformitarismo» basáncłose en que las catástrofes actualmente ocurren, y si ocurrieron en el pasado son, más bien, el ejemplo y no la contradicción de un principio de uniformidad.

\section{IMPLICACIONES DIDÁCTICAS}

¿Qué aporta el análisis de las ideas, modelos o teorías sobre las transformaciones geológicas de la Tierra que han estado vigentes en el pasado? ¿Qué utilidad didáctica tiene ese análisis? ¿Es posible extraer algunas orientaciones acerca de las dificultades que presenta su aprendizaje y de posibles vías para su tratamiento en la Educación Secundaria? Son éstos, algunos de los interrogantes que podemos formularnos.

La controversia Catastrofismo/Uniformitarismo es un excelente ejemplo de cómo se construye la Ciencia, de los titubeos, avances y ${ }_{i}$ retrocesos? que tienen lugar a lo largo de ese proceso; de que nunca hay un solo descubridor (Gagliardi y Giordan 1986), sino más bien contribuciones parciales. Así, se ha pretendido mostrar que el planteamiento que habitualmente se hace del Uniformitarismo/ Actualismo como una aportación personal de Lyell, que tendría en Hutton su precursor, es no sólo simplificador sino también inexacto.

La relación entre Ciencia y Sociedad está presente, a veces de manera determinante, a lo largo del debate sobre la interpretación de los cambios geológicos. En efecto, puede analizarse la influencia de las ideas religiosas en las teorías científicas (edad de la Tierra, diluvio, etc.); o de las ideas políticas. Así, se produjo un intento de apropiación de las teorías uniformitaristas (cn el momento en que alcanzaron su mayor prestigio) por la ideología liberal-burguesa partidaria de pequeños cambios graduales, frente a las posiciones catastrofistas que serían pretendidamente revolucionarias. 
Todo ello hace de la historia de esta controversia un buen recurso didáctico para su uso en el aula: bien haciendo el comentario de un texto científico escogido, bien estudiando el debate sostenido en un determinado momento histórico, o bien, en un contexto más amplio, analizanclo las interacciones sociales que tienen lugar.

Pero son, quizá, las consideraciones que se puedan realizar sobre la enseñanza y el aprendizaje de los cambios geológicos, lo que otorga un valor inapreciable al análisis de las dificultades conceptuales y de procedimientos que la comunidad científica ha encontrado para su construcción. Veamos algunas de estas consideraciones:

Los cambios que se producen en el relieve terrestre no son evidentes. En el presente trabajo se han señalado diversas interpretaciones de la evolución de la superficie terrestre, atendiendo a las diferencias en los tipos de procesos que describen o intentan explicar, a las relaciones que se establecen entre ellos, a las fuerzas que ponen en juego, a los ritmos con que ocurren y, sobre todo, a los procedimientos que se utìlizan para interpretarlos.

No son históricamente excepcionales las visiones «fijistas» (especialmente durante los siglos XVI y XVII pero también a lo largo del XVIII), que, sencillamente, han negado la existencia de cambios o que los han minimizado. Esta posición coincidiría con ideas o representaciones frecuentes en los alumnos (Pedrinaci 1987). Ello aconseja considerar el paso de concepciones estáticas a otras progresivamente más dinámicas como un proceso esencial que, de no producirse, bloquearía futuros avances.

El abandono de las posiciones "fijistas" para asumir perspectivas "movilistas" no parece que ocurra de una sola vez, afectando a la totalidad de los procesos. No todos los fenómenos geológicos exigen para su percepción y conceptualización las mismas operaciones intelectuales, ni presentarán, en consecuencia, los mismos obstáculos para su construcción. Así, no resulta extraño que sean los procesos geológicos más espectaculares y de efectos casi instantáneos, como los seísmos o las erupciones volcánicas, aquéllos que históricamente han sido mâs citados.

Los procesos erosivos también han sido frecuentemente descritos, si bien no siempre sufícientemente valorados. La dificultad de comprensión de la magnitud que pueden ađquirir sus efectos estaría relacionada con la noción da tiempo geológico.

La relación causa-efecto entre erosión y sedimentación. aunque dista de ser evidente, no parece encerrar una excesiva dificultad. Por ello, como puede verse al comienzo de este trabajo, la encontramos en algunas de las primeras descripciones geológicas que hacen los clásicos (véase, por ejemplo, Heródoto). Conviene, sin embargo, hacer constar que la dificultad aumenta para los procesos de sedimentación relacionados con las sustancias disueltas en el agua.

Mucha mayor resistencia parece haber ofrecido el concepto de diagénesis. En efecto, el paso de sedimento a roca sedimentaria es de aparición relativamente tardía e in- frecuente en la literatura geológica antigua. Buena parte de los problemas históricos para la interpretación deI origen de los fósiles tienen aquí su causa. Un motivo similar puede tener el recurso, tan habitual durante los sigios XV, XVI y XVII, a la existencia de la evirtus lapidifica" y el carácter mágico que se le otorga como elemento capaz de convertir en piedra sustancias de origen muy diverso.

Ello debe llevarnos, cuando menos, a no subestimar las dificultades que pueden tener los alumnos en la construcción de dicho concepto. La ausencia de la diagénesis se integra, por otra parte, dentro de una visión unidireccional de la dinámica geológica en la que faltarían los procesos constructivos.

La naturaleza de los materiales implicados (rocas) junto con los problemas de construcción del concepto de tiempo geológico parecen subyacer en la resistencia intuitiva al cambio (Pedrinaci 1992). Los eventos geológicos pueden convertirse por ello en unos recursos didácticos de primera magnitud, especialmente los eventos locales, por encima de los de escala planetaria referidos con anterioridad. En efecto, grandes inundaciones, coladas de barro que cortan una carretera o sepultan unas casas, playas que son desmanteladas por la erosión marina... son fenómenos que se producen con relativa frecuencia. Planteados como «estudio de casos», presentan algunas ventajas; de una parte, porque son cambios fácilmente constatables, al producirse efectos importantes en un corto margen de tiempo y, de otra, porque no sólo son apreciables los efectos sino que, con frecuencia, lo son también los agentes causantes.

Una vez aceptada, aunque sólo sea como hipotesis de trabajo, la existencia de cambios en el relieve terrestre, la naturaleza de los materiales puestos en juego y, especialmente, la magnitud de dichos cambios, parecen favorecer la tendencia a recurrir a fucrzas mágicas o a mitos. Es lo que podríamos denominar como propensión al catastrofismo.

De acuerdo con Pozo y otros (1991), en nuestros análisis causales tendemos a creer que existe una semejanza básica entre las causas y los efectos. Esa regla de semejanza causal adoptaría en este caso su perspectiva cuantitativa: «a grandes efectos, grandes causas», y si se desconocen dichas causas, se inventan (mitos) o se hace una extrapolación acrítica. La «propensión al catastrofismo» en su sentido inicial o acientífico vendría confirmada por su presencia entre las concepciones de los alumnos (Pedrinaci 1987) y en el saber común de numerosos pueblos desde la antigüiedad. Conviene destacar que no se trata, sólo, de una concepción que interpreta la realidad de una manera que hoy se considera inadecuada, sino que, en la medida en que no pretende realizar una interpretación racional, fundamentada o constatable, cortocircuita el razonamiento y se convierte en inhibidora de la progresión conceptual, de ahf́ su importancia.

Para finalizar, habría que destacar la importancia didáctica del Actualismo como método de análisis. Su uso facilita la comprensión de los procesos geológicos, la movilización de las concepciones estáticas y, en definitiva, el 
aprendizaje de Ia Geología. Su interés didáctico sería paralelo a la enorme potencialidad científica que ha mostrado a lo largo de la historia de la Geología. En efecto, las ciencias de la Tierra actualmente vigentes han sido construidas sobre el principio del Actualismo. Su potencialidad es tal que, habiendo sido la base del Uniformitarismo, ha posibilitado su revisión y la formulación del Neocatastrofismo.

\section{REFERENCIAS BHBLIOGRÁFICAS}

ALVARE7, W. et al., 1980. Extraterrestrial cause for de Creta ceous-Tertiary extinction, Science, 208. pp. 1095-1110.

ANGUITA, I*, 1988. Origen e Historia de la Tierra (Rueda: Madrid).

ARISTÓ'LLES.Les Meteorolologiques. (Trad. J. Tricot, 1955 , Librairie Philosophique. J. Vrin: París).

BERGGRFN, W. et aI., 1984. Catastrophes and Earth History. The new uniformitarism . (Princeton University Press: Princeton).

CAILLFUX, A., 1961. Histoire de la Géologie (Presses Universitaires de France: París). (Trad. N. Bastard, 1964, $\mathrm{His-}$ toria de la geología, Eudeba: Rivadavia).

CAPEL, H., 1987. Naturaleza y cultura en los origenes de la Geología española en Ilistoria de las Ciencias. (Consejo Superior de Investigaciones (ientificas : Madrid).

CARRASCOSA, J. y GLL, D., 1985. I a metodología de la superficialidad y el aprendizaje de las ciencias, Enseñanza de las Ciencias, Vol. 3, pp. 113-120.

CROMBIE, A.C., 1959. Augustine to Galileo (Trad. J. Bernia, Historia de la Ciencia: de San Agustín a Galileo, Alianza: Madrid).

DUHEM, P., 1959. Le système du monde (Hermann:París).

ELLENBERGER, F, 1988. Histoire de la Geologie (Trad, M. Rubi6, 1989, Historia de la Geología, Labor: Madrid).

GAGLIARDI, R, y GIORDAN, A., 1986. La historia de las ciencias: una herramienta para la enseñanza, Enseñanza de las Ciencias, Vol. 4, pp. 253-258.

GAGLIARDI, R, 1988. Como utilizar la historia de las ciencias en la enseñanza de las ciencias, Enseñanza de las Ciencias, Vol. 6, pp. 291-296.

GII, D. y CARRASCOSA, J., 1985. Science learning as a conceptual and mcthodological change, European Journal of Science Education, Vol. 7, pp. 231-236.

GOHAU, G., 1983. Idées anciennes sur la formation des montagnes, Cahiers d'Histoire et Philosophie des Sciences, 7.

HALI.AM, A. 1983. Great Geological Controversies. (Oxford University Press). (Trad. J. M. Fontboté, Grandes controversias geológicas, Labor: Barcelona).

HOOYKAAS, R., 1970. Continuité et discontinuité en Géologie et Biologie. (París).

IHSU, K., 1983. Actualistic Catastrophism. Sedimentology, Vol. 30, pp. 3-9.
KHUN, T. 1962. The Structure of Scientifi: Revolutions (University of Chicago Press). (A. Contin, La estructura de las revoluciones científicas, Fondo de Cultura Economica: México).

KIRCHER, A., 1989. El arca de Noé. (Trad. Atilano Martíncz, 1989, Octo: Madrid).

KITYYS, D., 1970. Teoría de la Geología, en C. Albritton (cd.) Filosofía de la Geología, (Compañía Ed. Continental: México).

McINTYRE, D., 1970. James Hutton y ta filosofía de la geologia, en C. Albritton (cd.), Filosofía de la Geología. (Compañía Ed. Continental; México).

OVIDIO, 1988. Metamorfosis (Trad. A. Ruiz de Flvira, Consejo Superior de Investigaciones Científicas: Madrid).

PEDRINACI, E., 1987. Representaciones de los alumnos sobre los cambios geologicos, Investigación en la Escuela, 2, pp. $65-74$.

PEDRINACI, E. 1991. Epistemología, Historia de la Ciencia y abejas. VIII Jornadas de Investigación en Ia Escuela. Universidad de Sevilla.

PEDRINACI, E. 1992. Fl concepto de Tiempo Geológico: uno perspectiva histórica. III Congreso Geológico de Españat. Universidad de Salamanca (en prensa).

SALTIEL, E. y VIFNNOT, L., 1985. ¿Qué aprendemos de las semejanzas entre las ideas históricas y el razonamiento espontánco de los alumnos, Enseñanza de las Ciencias, Vol. 3, pp. $137-144$.

SENECA, 1979. Cuestiones Naturales. (Trad. C. Codoñer, 1979, Consejo Superior de Investigaciones Científicas: Madrid).

SFQUEIROS, L., 1981. El método de los paradigmas de $K \mathrm{~W}$ interpela a las Ciencias Geológicas: notas para una Geología sin dogmas. Simposium Nac. Enserianza Geología, (Universidad Complutense: Madrid), pp. 437-444.

SEQUEIROS, L., 1990. Paleontología, Catástrofes y Extinciones en Masa, Razón y $F e, 1095$, pp. 54-62.

TATON, R. (ed.), 1964. Histoire genérale des sciences (4 vol). PUF: París, (Trad. Mistoria General de las Ciencias , $197 \mathrm{l}$, Destino: Barcelona).

VISR, J.A., 1990. Estratigrafia y Geología de Eventos. Discurso de ingreso en la Real Academia de Ciencias de Granada. 